\title{
ON ULTIMATE RUIN IN A DELAYED-CLAIMS RISK MODEL
}

\author{
KAM C. YUEN, ${ }^{* *}$ The University of Hong Kong \\ JUNYI GUO, ${ }^{* * *}$ Nankai University \\ KAI W. NG, ${ }^{*}$ The University of Hong Kong
}

\begin{abstract}
In this paper, we consider a risk model in which each main claim induces a delayed claim called a by-claim. The time of delay for the occurrence of a by-claim is assumed to be exponentially distributed. From martingale theory, an expression for the ultimate ruin probability can be derived using the Lundberg exponent of the associated nondelayed risk model. It can be shown that the Lundberg exponent of the proposed risk model is the same as that of the nondelayed one. Brownian motion approximations for ruin probabilities are also discussed.
\end{abstract}

Keywords: Brownian motion; by-claim; Lundberg exponent; main claim; martingale; ultimate ruin probability; weak convergence

2000 Mathematics Subject Classification: Primary 60J65; 62P05

Secondary 60H05; 60K99; 90A09

\section{Introduction}

In reality, insurance claims may be delayed due to various reasons. Risk models with this special feature have been discussed in the literature for some years. Waters and Papatriandafylou (1985) considered a discrete-time risk model allowing for delay in claims settlements and used martingale techniques to derive upper bounds for ruin probabilities. Boogaert and Haezendonck (1989) studied the mathematical properties of a liability process with settling delay within the framework of an economics environment.

In this paper, we assume that each main claim induces another type of claim, called a byclaim. The two types of claim have different distributions of severity. The time of occurrence of a by-claim is later than that of its main claim, and the time of delay for a by-claim is random. This kind of risk modeling may be of practical use. For instance, a serious motor accident causes different kinds of claim, such as car damage, injury, and death; some can be dealt with immediately while others need a random period of time to be settled.

The paper is organized as follows. In Section 2, we give a detailed description of the model. In Section 3, we use the martingale method to derive an expression for the ultimate ruin probability, which involves a nondelayed surplus process as well as its Lundberg exponent. In Section 4, we show that the Lundberg exponent of the proposed model with delayed claims is the same as that of the associated nondelayed risk model. Finally, in Section 5, we investigate the

Received 13 January 2004; revision received 13 July 2004.

* Postal address: Department of Statistics and Actuarial Science, The University of Hong Kong, Pokfulam Road, Hong Kong.

** Email address: kcyuen@hku.hk

*** Postal address: School of Mathematical Sciences and LPMC, Nankai University, Tianjin 300071, P. R. China. 
weak convergence of the aggregate claims process and derive Brownian motion approximations for ruin probabilities.

\section{The model}

Yuen and Guo (2001) used the method of probability-generating functions to derive ruin probabilities for the compound binomial model with discrete delay time for by-claims. In this paper, we consider a similar problem in the continuous-time setting. Specifically, we study various aspects of the compound Poisson model with delayed claims.

Let the aggregate main claims process be a compound Poisson process and let $N(t)$ be the corresponding Poisson claim number process, with intensity $\lambda$. Its jump times are denoted by $\left\{T_{i}, i=1,2, \ldots\right\}$ with $T_{0}=0$. The main claim amount random variables $\left\{X_{i}, i=1,2, \ldots\right\}$ follow a common distribution $F$ with mean $\mu_{F}$ and variance $\sigma_{F}^{2}$. In our model, if $X_{i}$ occurs at $T_{i}$, it will generate a by-claim, denoted by $Y_{i}$, occurring at time $T_{i}+W_{i}$, where $W_{i}$ is the random time of delay for $Y_{i}$. The by-claim amount random variables $\left\{Y_{i}, i=1,2, \ldots\right\}$ have a common distribution $G$ with mean $\mu_{G}$ and variance $\sigma_{G}^{2}$, while the delay times $\left\{W_{i}, i=1,2, \ldots\right\}$ are exponentially distributed with mean $\lambda_{1}^{-1}$. It is assumed that the $X_{i}, Y_{i}, T_{i}$, and $W_{i}$ are independent. All the random variables and random processes considered in this paper are defined on the probability space $(\Omega, \mathcal{F}, \mathrm{P})$.

In this setup, the surplus process takes on the form

$$
S(t)=u+c t-D(t)
$$

where $u$ is the initial surplus, $c$ is the rate of premium,

$$
D(t)=\sum_{i=1}^{N(t)} X_{i}+\sum_{i=1}^{\infty} Y_{i} \mathbf{1}\left(T_{i}+W_{i} \leq t\right),
$$

and $\mathbf{1}(\cdot)$ is the indicator function of the event $\{\cdot\}$. The associated by-claim number process is given by

$$
\bar{N}(t)=\sum_{i=1}^{\infty} \mathbf{1}\left(T_{i}+W_{i} \leq t\right) .
$$

It is obvious that the incorporation of the aggregate by-claims process complicates the analysis of surplus process (2.1).

Write the distribution of $T_{i}$ as $H_{T_{i}}$. The expectation of the aggregate by-claims at time $t$ is given by

$$
\begin{aligned}
\mathrm{E}\left[\sum_{i=1}^{\infty} Y_{i} \mathbf{1}\left(T_{i}+W_{i} \leq t\right)\right] & =\sum_{i=1}^{\infty} \mu_{G} \mathrm{P}\left(T_{i}+W_{i} \leq t\right) \\
& =\mu_{G} \int_{0}^{t} \sum_{i=1}^{\infty} H_{T_{i}}(t-x) \lambda_{1} \mathrm{e}^{-\lambda_{1} x} \mathrm{~d} x \\
& =\mu_{G} \int_{0}^{t} \lambda(t-x) \lambda_{1} \mathrm{e}^{-\lambda_{1} x} \mathrm{~d} x \\
& =\lambda \mu_{G}\left(t-\frac{1}{\lambda_{1}}+\frac{1}{\lambda_{1}} \mathrm{e}^{-\lambda_{1} t}\right) .
\end{aligned}
$$


Hence, to satisfy the positive safety loading condition, we may simply assume that $c>\lambda\left(\mu_{F}+\right.$ $\left.\mu_{G}\right)$. Let $T=\inf \{t: S(t)<0\}$ be the time of ruin for surplus process (2.1). Then, the ultimate ruin probability for $(2.1)$ is given by $\psi(u)=\mathrm{P}(T<\infty)$.

\section{A martingale approach for the ultimate ruin probability}

In this section, we use martingale techniques to derive an expression for the ultimate ruin probability for $S(t)$. Specifically, the ruin probability can be expressed in terms of a slightly modified surplus process and its Lundberg exponent.

Define a modification of $S(t)$ by

$$
S^{*}(t)=u+c t-D^{*}(t)
$$

where $D^{*}(t)=\sum_{i=1}^{N(t)}\left(X_{i}+Y_{i}\right)$. Note that (3.1) is a compound Poisson process (a nondelayed risk model) with $X_{i}+Y_{i}$ being the claim amount for the $i$ th claim. Let $\mathcal{F}_{t}=\sigma\{S(u), u \leq t\}$ and $\mathcal{F}_{t}^{*}=\sigma\left\{S^{*}(u), u \leq t\right\}$ be the natural filtrations of $S(t)$ and $S^{*}(t)$, respectively. The Lundberg exponent $R^{*}$ for $S^{*}(t)$ is defined to be the positive solution of $\mathrm{E}\left[\mathrm{e}^{r(X+Y)}\right]=1+\lambda^{-1} c r$. From classical ruin theory, we know that

$$
M^{*}(t)=\mathrm{e}^{-R^{*} S^{*}(t)}
$$

is a martingale relative to the filtration $\mathcal{F}_{t}{ }^{*}$. Now, consider two other filtrations given by

$$
\mathcal{g}_{t}^{*}=\mathcal{F}_{t}^{* X} \vee \mathcal{F}_{t}^{* Y} \text { and } \mathcal{g}_{t}=\mathcal{g}_{t}^{*} \vee \sigma\left\{W_{1}, W_{2}, \ldots\right\}
$$

where $\mathcal{F}_{t}^{* X}$ is the natural filtration of $\sum_{i=1}^{N(t)} X_{i}$ and $\mathcal{F}_{t}^{* Y}$ is the natural filtration of $\sum_{i=1}^{N(t)} Y_{i}$. It is obvious that $g_{t}^{*} \subset g_{t}$ and that $M^{*}(t)$ is a $g_{t}^{*}$ martingale. Moreover, we make the following proposition.

Proposition 3.1. For the time of ruin $T$ and the filtrations $\mathcal{F}_{t}, \mathcal{F}_{t}^{*}, g_{t}, g_{t}^{*}$, and $M^{*}(t)$, the following statements hold.

(a) (i) $\mathcal{F}_{t}^{*} \subset g_{t}^{*}$ and (ii) $\mathcal{F}_{t} \subset g_{t}$.

(b) $M^{*}(t)$ is a martingale relative to filtration $g_{t}$.

(c) $T$ is a stopping time relative to $\mathcal{F}_{t}$ and $g_{t}$.

Proof. Since $D^{*}(t)=\sum_{i=1}^{N(t)} X_{i}+\sum_{i=1}^{N(t)} Y_{i}$, part (a)(i) follows immediately. Write

$$
W_{i}^{(k)}=\sum_{j=0}^{\infty} \frac{j+1}{k} \mathbf{1}\left(\frac{j}{k}<W_{i} \leq \frac{j+1}{k}\right)
$$

and

$$
D_{Y}^{(k)}(t)=\sum_{i=1}^{N(t)} Y_{i} \mathbf{1}\left(T_{i}+W_{i}^{(k)} \leq t\right)
$$

Then, we have

$$
\lim _{k \rightarrow \infty} W_{i}^{(k)}=W_{i} \quad \text { and } \quad \lim _{k \rightarrow \infty} D_{Y}^{(k)}(t)=\sum_{i=1}^{N(t)} Y_{i} \mathbf{1}\left(T_{i}+W_{i} \leq t\right) .
$$


Thus, to prove part (a)(ii) it suffices to show that $D_{Y}^{(k)}(t)$ is $g_{t}$ measurable. It can easily be shown that

$$
\begin{aligned}
& \mathbf{1}(N(t)=n) D_{Y}^{(k)}(t) \\
& =\sum\left(\mathbf{1}(N(t)=n)\left(\prod_{i=1}^{n} \mathbf{1}\left(\frac{j_{i}}{k}<W_{i} \leq \frac{j_{i}+1}{k}\right)\right) \sum_{i=1}^{n} Y_{i} \mathbf{1}\left(T_{i} \leq t-\frac{j_{i}+1}{k}\right)\right),
\end{aligned}
$$

where the first summation on the right-hand side is over all $j_{i}=0,1, \ldots$ for $i=1, \ldots, n$. Notice that

$$
\begin{aligned}
\mathbf{1}(N(t)=n)\left(\prod_{i=1}^{n} \mathbf{1}\left(\frac{j_{i}}{k}<W_{i} \leq \frac{j_{i}+1}{k}\right)\right) \sum_{i=1}^{n} Y_{i} \mathbf{1}\left(T_{i} \leq t-\frac{j_{i}+1}{k}\right) \\
=\mathbf{1}(N(t)=n)\left(\prod_{i=1}^{n} \mathbf{1}\left(\frac{j_{i}}{k}<W_{i} \leq \frac{j_{i}+1}{k}\right)\right) \sum_{i=1}^{n} Y_{i} \mathbf{1}\left(N\left(t-\frac{j_{i}+1}{k}\right) \geq i\right) .
\end{aligned}
$$

Equalities (3.2) and (3.3) imply that $\mathbf{1}(N(t)=n) D_{Y}^{(k)}(t)$ is $g_{t}$ measurable, because the product term on both sides of (3.3) is $\sigma\left\{W_{1}, W_{2}, \ldots\right\}$ measurable.

For part (b), we must prove that $\mathrm{E}\left[M^{*}(t) \mid g_{s}\right]=M^{*}(s)$ almost surely for $t \geq s$, i.e. that, for each $G \in g_{s}, \int_{G} M^{*}(t) \mathrm{dP}=\int_{G} M^{*}(s) \mathrm{d} \mathrm{P}$. Because of the structure of $g_{s}$, this is equivalent to proving that $G=K_{1} \cap K_{2}$, where $K_{1} \in g_{t}^{*}$ and $K_{2} \in \sigma\left\{W_{1}, W_{2}, \ldots, W_{n}\right\}$. Since $K_{1}$ and $K_{2}$ are independent, $K_{2}$ is independent of $M^{*}(t)$. Therefore,

$$
\int_{K_{1} \cap K_{2}} M^{*}(t) \mathrm{d} \mathrm{P}=\mathrm{P}\left(K_{2}\right) \int_{K_{1}} M^{*}(t) \mathrm{d} \mathrm{P}=\mathrm{P}\left(K_{2}\right) \int_{K_{1}} M^{*}(s) \mathrm{d} \mathrm{P}=\int_{K_{1} \cap K_{2}} M^{*}(s) \mathrm{d} \mathrm{P},
$$

because $M^{*}(t)$ is a $g_{t}^{*}$ martingale.

Part (c) follows from part (a)(ii) and the definition of $T$.

We now present the main result of this section, which states that $S^{*}(t)$ and its Lundberg exponent $R^{*}$ play an important role in studying the ruin probability for $S(t)$.

Theorem 3.1. The ultimate ruin probability for $S(t)$ can be expressed as

$$
\psi(u)=\frac{\mathrm{e}^{-R^{*} u}}{\mathrm{E}\left[\mathrm{e}^{-R^{*} S^{*}(T)} \mid T<\infty\right]},
$$

where $T$ is the time of ruin for $S(t)$.

Proof. Since $M^{*}(t)$ is a martingale and T is a stopping time, it follows from the optional stopping theorem that

$$
\begin{aligned}
\mathrm{e}^{-R^{*} u}=M^{*}(0) & =\mathrm{E}\left[M^{*}(t \wedge T)\right] \\
& =\mathrm{E}\left[M^{*}(t \wedge T) \mid T \leq t\right] \mathrm{P}(T \leq t)+\mathrm{E}\left[M^{*}(t \wedge T) \mid T>t\right] \mathrm{P}(T>t)
\end{aligned}
$$

Hence, the theorem is proved if

$$
\mathrm{E}\left[M^{*}(t \wedge T) \mid T>t\right] \mathrm{P}(T>t) \rightarrow 0 \quad \text { as } t \rightarrow \infty .
$$


The following two expectations will be used to prove (3.4):

$$
Z_{1}\left(R^{*}\right)=\mathrm{E}\left[S(t) \mathrm{e}^{R^{*}\left(D^{*}(t)-c t\right)}\right] \quad \text { and } Z_{2}\left(R^{*}\right)=\mathrm{E}\left[S(t)^{2} \mathrm{e}^{R^{*}\left(D^{*}(t)-c t\right)}\right] .
$$

We first consider $Z_{1}\left(R^{*}\right)$. Let

$$
Z_{1}\left(R^{*}\right)=u+c t-Z_{11}\left(R^{*}\right)-Z_{12}\left(R^{*}\right)
$$

where

$$
\begin{aligned}
& Z_{11}\left(R^{*}\right)=\mathrm{E}\left[\sum_{i=1}^{N(t)} X_{i} \mathrm{e}^{R^{*}\left(D^{*}(t)-c t\right)}\right], \\
& Z_{12}\left(R^{*}\right)=\mathrm{E}\left[\sum_{i=1}^{\infty} Y_{i} \mathbf{1}\left(T_{i}+W_{i} \leq t\right) \mathrm{e}^{R^{*}\left(D^{*}(t)-c t\right)}\right],
\end{aligned}
$$

and define $h_{X}(r)=\mathrm{E}\left[\mathrm{e}^{r X}\right]-1, h_{Y}(r)=\mathrm{E}\left[\mathrm{e}^{r Y}\right]-1$, and $h(r)=\mathrm{E}\left[\mathrm{e}^{r(X+Y)}\right]-1$. Then,

$$
\begin{aligned}
Z_{11}(r) & =\mathrm{e}^{-r c t} \sum_{n=1}^{\infty}\left(\frac{(\lambda t)^{n} \mathrm{e}^{-\lambda t}}{n !} \sum_{i=1}^{n} \mathrm{E}\left[X_{i} e^{r X_{i}} \exp \left\{r \sum_{j \leq n, j \neq i} X_{j}\right\} \exp \left\{r \sum_{i=1}^{n} Y_{i}\right\}\right]\right) \\
& =\mathrm{e}^{-r c t} \sum_{n=1}^{\infty}\left(\frac{(\lambda t)^{n} \mathrm{e}^{-\lambda t}}{n !}\left(h_{Y}(r)+1\right)^{n}\left(h_{X}(r)+1\right)^{n-1} \sum_{i=1}^{n} \mathrm{E}\left[X_{i} \mathrm{e}^{r X_{i}}\right]\right) .
\end{aligned}
$$

Since $\mathrm{E}\left[X_{i} e^{r X_{i}}\right]=h_{X}^{\prime}(r), h(r)+1=\left(h_{X}(r)+1\right)\left(h_{Y}(r)+1\right)$, and $\lambda h\left(R^{*}\right)-c R^{*}=0$, where a prime denotes differentiation, $Z_{11}\left(R^{*}\right)$ becomes

$$
Z_{11}\left(R^{*}\right)=\left(h_{Y}\left(R^{*}\right)+1\right) h_{X}^{\prime}\left(R^{*}\right) \lambda t .
$$

We also have

$$
\begin{aligned}
Z_{12}(r) & =\mathrm{e}^{-r c t} \sum_{n=1}^{\infty} \mathrm{E}\left[Y_{n} \mathbf{1}\left(T_{n}+W_{n} \leq t\right) \mathrm{e}^{r D^{*}(t)}\right] \\
& =\mathrm{e}^{-r c t} \sum_{n=1}^{\infty} \int_{0}^{\infty} \mathrm{E}\left[Y_{n} \mathbf{1}\left(T_{n}+W_{n} \leq t\right) \mathrm{e}^{r D^{*}(t)} \mid W_{n}=s\right] \mathrm{P}\left(W_{n}=s\right) \mathrm{d} s \\
& =\mathrm{e}^{-r c t} \sum_{n=1}^{\infty} \int_{0}^{t} \lambda_{1} \mathrm{e}^{-\lambda_{1} s} \mathrm{E}\left[Y_{n} \mathbf{1}\left(T_{n} \leq t-s\right) \mathrm{e}^{r D^{*}(t)}\right] \mathrm{d} s \\
& =\mathrm{e}^{-r c t} \int_{0}^{t} \lambda_{1} \mathrm{e}^{-\lambda_{1} s} \mathrm{E}\left[\mathrm{e}^{r D^{*}(t)} \sum_{n=1}^{\infty} Y_{n} \mathbf{1}\left(T_{n} \leq t-s\right)\right] \mathrm{d} s \\
& =\mathrm{e}^{-r c t} \int_{0}^{t} \lambda_{1} \mathrm{e}^{-\lambda_{1} s} \mathrm{E}\left[\mathrm{e}^{r D^{*}(t)} \sum_{n=1}^{N(t-s)} Y_{n}\right] \mathrm{d} s \\
& =\mathrm{e}^{-r c t} \int_{0}^{t} \lambda_{1} \mathrm{e}^{-\lambda_{1} s} \mathrm{E}\left[\mathrm{e}^{r D^{*}(t-s)} \exp \left\{r\left(D^{*}(t)-D^{*}(t-s)\right)\right\} \sum_{n=1}^{N(t-s)} Y_{n}\right] \mathrm{d} s \\
& =\int_{0}^{t} \lambda_{1} \mathrm{e}^{-\lambda_{1} s} \mathrm{E}\left[\exp \left\{r\left(D^{*}(t-s)-c(t-s)\right)\right\} \sum_{n=1}^{N(t-s)} Y_{n}\right] \mathrm{E}\left[e^{r\left(D^{*}(s)-c s\right)}\right] \mathrm{d} s .
\end{aligned}
$$


From (3.6) and the fact that $\mathrm{E}\left[\mathrm{e}^{R^{*}\left(D^{*}(s)-c s\right)}\right]=1$ for all $s, Z_{12}\left(R^{*}\right)$ can be written as

$$
\begin{aligned}
Z_{12}\left(R^{*}\right) & =\int_{0}^{t} \lambda_{1} \mathrm{e}^{-\lambda_{1} s}\left(h_{X}\left(R^{*}\right)+1\right) h_{Y}^{\prime}\left(R^{*}\right) \lambda(t-s) \mathrm{d} s \\
& =\left(h_{X}\left(R^{*}\right)+1\right) h_{Y}^{\prime}\left(R^{*}\right)\left(\lambda t-\frac{\lambda}{\lambda_{1}}\left(1-\mathrm{e}^{-\lambda_{1} t}\right)\right) .
\end{aligned}
$$

Then, substituting the derived forms of $Z_{11}\left(R^{*}\right)$ and $Z_{12}\left(R^{*}\right)$ into (3.5), we obtain

$$
Z_{1}\left(R^{*}\right)=u+c t-\lambda t h^{\prime}\left(R^{*}\right)+\frac{\lambda}{\lambda_{1}}\left(1-\mathrm{e}^{-\lambda_{1} t}\right)\left(h_{X}\left(R^{*}\right)+1\right) h_{Y}^{\prime}\left(R^{*}\right) .
$$

We next look for an upper bound for $Z_{2}\left(R^{*}\right)$. First, write

$$
Z_{2}(r)=(u+c t)^{2}-2(u+c t) Z_{21}(r)+Z_{22}(r)+Z_{23}(r)+2 Z_{24}(r),
$$

where

$$
\begin{aligned}
& Z_{21}(r)=\mathrm{E}\left[\left(\sum_{i=1}^{N(t)} X_{i}+\sum_{i=1}^{\infty} Y_{i} \mathbf{1}\left(T_{i}+W_{i} \leq t\right)\right) \mathrm{e}^{r\left(D^{*}(t)-c t\right)}\right], \\
& Z_{22}(r)=\mathrm{E}\left[\left(\sum_{i=1}^{N(t)} X_{i}\right)^{2} \mathrm{e}^{r\left(D^{*}(t)-c t\right)}\right], \\
& Z_{23}(r)=\mathrm{E}\left[\left(\sum_{i=1}^{\infty} Y_{i} \mathbf{1}\left(T_{i}+W_{i} \leq t\right)\right)^{2} \mathrm{e}^{r\left(D^{*}(t)-c t\right)}\right], \\
& Z_{24}(r)=\mathrm{E}\left[\left(\sum_{i=1}^{N(t)} X_{i}\right)\left(\sum_{i=1}^{\infty} Y_{i} \mathbf{1}\left(T_{i}+W_{i} \leq t\right)\right) \mathrm{e}^{r\left(D^{*}(t)-c t\right)}\right] .
\end{aligned}
$$

Note that $Z_{21}\left(R^{*}\right)=Z_{11}\left(R^{*}\right)+Z_{12}\left(R^{*}\right)$, i.e.

$$
Z_{21}\left(R^{*}\right)=\lambda t h^{\prime}\left(R^{*}\right)-\frac{\lambda}{\lambda_{1}}\left(1-\mathrm{e}^{-\lambda_{1} t}\right) h_{Y}^{\prime}\left(R^{*}\right)\left(h_{X}\left(R^{*}\right)+1\right) .
$$

To evaluate $Z_{22}(r)$, we need

$$
\begin{aligned}
H(r) & =\mathrm{E}\left[\left(\sum_{i=1}^{n} X_{i}\right)^{2} \exp \left\{r \sum_{i=1}^{n} X_{i}\right\}\right] \\
& =\left[\left(h_{X}(r)+1\right)^{n}\right]^{\prime \prime} \\
& =n\left(h_{X}(r)+1\right)^{n-2}\left((n-1)\left(h_{X}^{\prime}(r)\right)^{2}+\left(h_{X}(r)+1\right) h_{X}^{\prime \prime}(r)\right),
\end{aligned}
$$

which implies that

$$
\begin{aligned}
Z_{22}(r) & =\sum_{n=0}^{\infty} \frac{(\lambda t)^{n} \mathrm{e}^{-\lambda t}}{n !} \mathrm{E}\left[\left(\sum_{i=1}^{n} X_{i}\right)^{2} \mathrm{e}^{r\left(D^{*}(t)-c t\right)}\right] \\
& =\mathrm{e}^{-r c t} \sum_{n=1}^{\infty} \frac{(\lambda t)^{n} \mathrm{e}^{-\lambda t}}{n !}\left(h_{Y}(r)+1\right)^{n} H(r) .
\end{aligned}
$$


After some algebraic manipulation, we obtain

$$
\begin{aligned}
Z_{22}\left(R^{*}\right) & =\exp \left\{t\left(\lambda h\left(R^{*}\right)-c R^{*}\right)\right\} \lambda t\left(h_{Y}\left(R^{*}\right)+1\right)\left(\lambda t\left(h_{Y}\left(R^{*}\right)+1\right)\left(h_{X}^{\prime}\left(R^{*}\right)\right)^{2}+h_{X}^{\prime \prime}\left(R^{*}\right)\right) \\
& =\lambda t\left(h_{Y}\left(R^{*}\right)+1\right)\left(\lambda t\left(h_{Y}\left(R^{*}\right)+1\right)\left(h_{X}^{\prime}\left(R^{*}\right)\right)^{2}+h_{X}^{\prime \prime}\left(R^{*}\right)\right)
\end{aligned}
$$

Similarly, it can be shown that

$$
\begin{aligned}
Z_{23}\left(R^{*}\right) & \leq \mathrm{E}\left[\left(\sum_{i=1}^{N(t)} Y_{i}\right)^{2} \mathrm{e}^{R^{*}\left(D^{*}(t)-c t\right)}\right] \\
& =\lambda t\left(h_{X}\left(R^{*}\right)+1\right)\left(\lambda t\left(h_{X}\left(R^{*}\right)+1\right)\left(h_{Y}^{\prime}\left(R^{*}\right)\right)^{2}+h_{Y}^{\prime \prime}\left(R^{*}\right)\right) .
\end{aligned}
$$

For the last term on the right-hand side of (3.8), we obtain

$$
\begin{aligned}
Z_{24}\left(R^{*}\right) & \leq \mathrm{E}\left[\left(\sum_{i=1}^{N(t)} X_{i}\right)\left(\sum_{i=1}^{N(t)} Y_{i}\right) \mathrm{e}^{R^{*}\left(D^{*}(t)-c t\right)}\right] \\
& =\mathrm{e}^{-R^{*} c t-\lambda t} \sum_{n=0}^{\infty} \frac{(\lambda t)^{n}}{n !} \mathrm{E}\left[\sum_{i=1}^{n} X_{i} \exp \left\{R^{*} \sum_{i=1}^{n} X_{i}\right\} \sum_{i=1}^{n} Y_{i} \exp \left\{R^{*} \sum_{i=1}^{n} Y_{i}\right\}\right] \\
& =\mathrm{e}^{-R^{*} c t-\lambda t} \sum_{n=0}^{\infty} \frac{(\lambda t)^{n}}{n !}\left[\left(h_{X}(r)+1\right)^{n}\right]_{r=R^{*}}^{\prime}\left[\left(h_{Y}(r)+1\right)^{n}\right]_{r=R^{*}}^{\prime} \\
& =\mathrm{e}^{-R^{*} c t-\lambda t} h_{X}^{\prime}\left(R^{*}\right) h_{Y}^{\prime}\left(R^{*}\right) \sum_{n=1}^{\infty} \frac{(\lambda t)^{n}}{(n-1) !} n\left(h\left(R^{*}\right)+1\right)^{n-1} \\
& =\mathrm{e}^{-R^{*} c t-\lambda t} h_{X}^{\prime}\left(R^{*}\right) h_{Y}^{\prime}\left(R^{*}\right) \lambda t \mathrm{e}^{\lambda t\left(h\left(R^{*}\right)+1\right)}\left(1+\lambda t\left(h\left(R^{*}\right)+1\right)\right) \\
& =h_{X}^{\prime}\left(R^{*}\right) h_{Y}^{\prime}\left(R^{*}\right) \lambda t\left(1+\lambda t\left(h\left(R^{*}\right)+1\right)\right) .
\end{aligned}
$$

Substituting the derived forms of $Z_{21}\left(R^{*}\right), Z_{22}\left(R^{*}\right), Z_{23}\left(R^{*}\right)$, and $Z_{24}\left(R^{*}\right)$ into (3.8) gives

$$
\begin{aligned}
Z_{2}\left(R^{*}\right) \leq & (u+c t)^{2}-2(u+c t)\left(\lambda t h^{\prime}\left(R^{*}\right)-\frac{\lambda}{\lambda_{1}}\left(1-\mathrm{e}^{-\lambda_{1} t}\right) h_{Y}^{\prime}\left(R^{*}\right)\left(h_{X}\left(R^{*}\right)+1\right)\right) \\
& +\lambda t\left(h_{Y}\left(R^{*}\right)+1\right)\left(\lambda t\left(h_{Y}\left(R^{*}\right)+1\right)\left(h_{X}^{\prime}\left(R^{*}\right)\right)^{2}+h_{X}^{\prime \prime}\left(R^{*}\right)\right) \\
& +\lambda t\left(h_{X}\left(R^{*}\right)+1\right)\left(\lambda t\left(h_{X}\left(R^{*}\right)+1\right)\left(h_{Y}^{\prime}\left(R^{*}\right)\right)^{2}+h_{Y}^{\prime \prime}\left(R^{*}\right)\right) \\
& +2 h_{X}^{\prime}\left(R^{*}\right) h_{Y}^{\prime}\left(R^{*}\right) \lambda t\left(1+\lambda t\left(h\left(R^{*}\right)+1\right)\right) .
\end{aligned}
$$

The proof of (3.4) is as follows. Note that

$$
\mathrm{E}\left[M^{*}(t \wedge T) \mid T>t\right] \mathrm{P}(T>t)=\int_{T>t} M^{*}(t) \mathrm{dP} .
$$


It is clear that if $T>t$ then $S(t) \geq 0$. Since $c-\lambda h^{\prime}\left(R^{*}\right)<0$, it is easily seen from (3.7) that $Z_{1}\left(R^{*}\right)<0$ for large $t$. Therefore,

$$
\begin{aligned}
\int_{T>t} M^{*}(t) \mathrm{d} \mathrm{P} & \leq \int_{S(t) \geq 0} M^{*}(t) \mathrm{dP} \\
& \leq \int_{S(t) \geq Z_{1}\left(R^{*}\right) / 2} M^{*}(t) \mathrm{d} \mathrm{P} \\
& \leq \int_{\left|S(t)-Z_{1}\left(R^{*}\right)\right| \geq-Z_{1}\left(R^{*}\right) / 2} M^{*}(t) \mathrm{dP} \\
& \leq \int_{\left|S(t)-Z_{1}\left(R^{*}\right)\right| \geq-Z_{1}\left(R^{*}\right) / 2} \frac{4 \mathrm{e}^{-R^{*} S^{*}(t)}\left(S(t)-Z_{1}\left(R^{*}\right)^{2}\right)}{Z_{1}\left(R^{*}\right)^{2}} \mathrm{dP} \\
& \leq \frac{4 \mathrm{e}^{-R^{*} u}\left(Z_{2}\left(R^{*}\right)-Z_{1}\left(R^{*}\right)^{2}\right)}{Z_{1}\left(R^{*}\right)^{2}} .
\end{aligned}
$$

Making use of (3.7) and (3.9), we find that

$$
\begin{aligned}
Z_{2}\left(R^{*}\right)-Z_{1}\left(R^{*}\right)^{2} \leq & 2 \lambda t h^{\prime}\left(R^{*}\right) \frac{\lambda}{\lambda_{1}}\left(1-\mathrm{e}^{-\lambda_{1} t}\right) h_{Y}^{\prime}\left(R^{*}\right)\left(h_{X}\left(R^{*}\right)+1\right) \\
& +\lambda t h^{\prime \prime}(R)-\left(\frac{\lambda}{\lambda_{1}}\left(1-\mathrm{e}^{-\lambda_{1} t}\right) h_{Y}^{\prime}\left(R^{*}\right)\left(h_{X}\left(R^{*}\right)+1\right)\right)^{2} .
\end{aligned}
$$

Hence, the numerator on the right-hand side of (3.10) is of order $t$ and $Z_{1}\left(R^{*}\right)^{2}$ is of order $t^{2}$. We conclude that

$$
\mathrm{E}\left[M^{*}(t \wedge T) \mid T>t\right] \mathrm{P}(T>t)=\int_{T>t} M^{*}(t) \mathrm{dP} \rightarrow 0
$$

as $t \rightarrow \infty$.

\section{Lundberg exponent}

In this section, we show that $R^{*}$ is also the Lundberg exponent for model (2.1). Brémaud (2000) also discussed such an issue for the Poisson shot noise delayed-claims model.

Since the ruin probability for model (2.1) is smaller than that for the nondelayed model, we have $\psi(u) \leq \mathrm{e}^{-R^{*} u}$. To verify that $R^{*}$ is the Lundberg exponent, we must calculate $\mathrm{E}\left[\mathrm{e}^{r D(t)}\right]$. For $N(t)=k$, it is well known that the random vector $\left(T_{1}, T_{2}, \ldots, T_{k}\right)$ has the same distribution as the order statistics of $k$ independent and identically distributed uniform $[0, t]$ random variables. Furthermore, $N(t), X_{i}, Y_{i}$, and $W_{i}$ are independent. Thus, we have

$$
\begin{aligned}
\mathrm{E}\left[\mathrm{e}^{r D(t)}\right] & =\sum_{k=0}^{\infty} \mathrm{e}^{-\lambda t} \frac{(\lambda t)^{k}}{k !} \frac{k !}{t^{k}} \int_{0}^{t} \mathrm{~d} s_{1} \int_{s_{1}}^{t} \mathrm{~d} s_{2} \cdots \int_{s_{k-1}}^{t} \mathrm{~d} s_{k} \prod_{i=1}^{k} \mathrm{E}\left[\exp \left\{r\left(X_{i}+Y_{i} \mathbf{1}\left(s_{i}+W_{i} \leq t\right)\right)\right\}\right] \\
& =\mathrm{e}^{-\lambda t} \sum_{k=0}^{\infty} \frac{\lambda^{k}}{k !}\left(\int_{0}^{t} \mathrm{E}[\exp \{r(X+Y \mathbf{1}(W \leq s))\}] \mathrm{d} s\right)^{k} \\
& =\exp \left\{-\lambda t\left(1-t^{-1} \int_{0}^{t} \mathrm{E}[\exp \{r(X+Y \mathbf{1}(W \leq s))\}] \mathrm{d} s\right)\right\}
\end{aligned}
$$

where $(X, Y, W)$ is the generic random vector of $\left(X_{i}, Y_{i}, W_{i}\right)$. 
Now let $g(r)=\lim _{t \rightarrow \infty} t^{-1} \ln \mathrm{E}\left[\mathrm{e}^{r(D(t)-c t)}\right]$. It can easily be shown that

$$
\begin{aligned}
g(r) & =\lambda \mathrm{E}\left[\mathrm{e}^{r(X+Y)}-1\right]-c r \\
& =\lambda r \int_{0}^{\infty} \mathrm{e}^{r x}(1-F * G(x)) \mathrm{d} x-c r,
\end{aligned}
$$

where $F * G$ represents the convolution of $F$ and $G$. On the other hand, the results of Duffield and O'Connell (1995) give

$$
\lim _{u \rightarrow \infty} \frac{1}{u} \ln \psi(u)=-R,
$$

where $R=\sup \{r: g(r) \leq 0\}$. It follows from (4.1) that $R$ is exactly the Lundberg exponent of the nondelayed risk model (3.1). Therefore, $R=R^{*}$.

\section{Approximations for ruin probabilities}

This section is devoted to deriving Brownian motion approximations for ruin probabilities for model (2.1). This kind of approximation for the compound Poisson model can be found in Grandell (1977), (1978) and Iglehart (1969). Although the by-claim number process $\bar{N}(t)$ is neither Poisson nor renewal, we are still able to extend the classical results to the proposed model.

Define $\kappa^{2}=\sigma_{F}^{2}+\sigma_{G}^{2}+\left(\mu_{F}+\mu_{G}\right)^{2}$. The mean and variance of $D^{*}(t)$ are $\lambda t\left(\mu_{F}+\mu_{G}\right)$ and $\lambda t \kappa^{2}$, respectively. The following theorem states that, asymptotically, $D(t)$ has the same mean and standard deviation, and that the delay time distribution does not come into play in the limit.

Theorem 5.1. As $t \rightarrow \infty$,

$$
U(t)=\frac{D(t)-\lambda t\left(\mu_{F}+\mu_{G}\right)}{\left(\lambda t \kappa^{2}\right)^{1 / 2}} \stackrel{\mathrm{D}}{\rightarrow} N(0,1),
$$

where $\stackrel{\mathrm{D}}{\rightarrow}$ ' stands for convergence in distribution and $N(0,1)$ is a standard normal random variable. As a result, $(\lambda t)^{-1 / 2}(\bar{N}(t)-\lambda t) \stackrel{\mathrm{D}}{\rightarrow} N(0,1)$.

Proof. It is well known that

$$
\frac{D^{*}(t)-\lambda t\left(\mu_{F}+\mu_{G}\right)}{\left(\lambda t \kappa^{2}\right)^{1 / 2}} \stackrel{\mathrm{D}}{\rightarrow} N(0,1) .
$$

From (2.2), we find that

$$
\begin{aligned}
\mathrm{E}\left[\frac{D^{*}(t)-\lambda t\left(\mu_{F}+\mu_{G}\right)}{\left(\lambda t \kappa^{2}\right)^{1 / 2}}-U(t)\right] & =\mathrm{E}\left[\frac{\sum_{i=1}^{N(t)} Y_{i}-\sum_{i=1}^{\infty} Y_{i} \mathbf{1}\left(T_{i}+S_{i} \leq t\right)}{\left(\lambda t \kappa^{2}\right)^{1 / 2}}\right] \\
& =\frac{\lambda \mu_{G}\left(1-\mathrm{e}^{-\lambda_{1} t}\right)}{\lambda_{1}\left(\lambda t \kappa^{2}\right)^{1 / 2}} \\
& \rightarrow 0
\end{aligned}
$$

as $t \rightarrow \infty$. Hence,

$$
\frac{D^{*}(t)-\lambda t\left(\mu_{F}+\mu_{G}\right)}{\left(\lambda t \kappa^{2}\right)^{1 / 2}}-U(t) \rightarrow 0
$$


in probability. This implies the weak convergence of $U(t)$. If we let $X_{i}=0$ and $Y_{i}=1$, the convergence of the by-claim number process is simply a special case of the convergence of $U(t)$.

Now define

$$
U_{n}(t)=\frac{D(n t)-\lambda n t\left(\mu_{F}+\mu_{G}\right)}{\left(\lambda n \kappa^{2}\right)^{1 / 2}} .
$$

To obtain the desired Brownian motion approximations, we must establish the weak convergence of $U_{n}(t)$. The symbol ' $\stackrel{\mathrm{W}}{\rightarrow}$ ' represents weak convergence for stochastic processes.

Theorem 5.2. For constant $t, U_{n}(t) \stackrel{\mathrm{W}}{\rightarrow} B(t)$ as $n \rightarrow \infty$, where $B(t)$ is a standard Brownian motion.

Proof. To prove the theorem, we need to prove (i) the convergence of the finite-dimensional distributions of $U_{n}(t)$ and (ii) the uniform tightness of $U_{n}(t)$, i.e. that, for every $\varepsilon>0$,

$$
\lim _{c \rightarrow 0} \limsup _{n \rightarrow \infty} \Delta_{J_{1}}^{\mathrm{P}}\left(c, U_{n}(t), \varepsilon\right)=0,
$$

where

$$
\Delta_{J_{1}}^{\mathrm{P}}\left(c, U_{n}(t), \varepsilon\right)=\sup _{t_{1}<t<t_{2}} \min \left(\mathrm{P}\left(\left|U_{n}(t)-U_{n}\left(t_{1}\right)\right|>\varepsilon\right), \mathrm{P}\left(\left|U_{n}\left(t_{2}\right)-U_{n}(t)\right|>\varepsilon\right)\right),
$$

with $t_{1} \geq t-c$ and $t_{2} \leq t+c$. Note that, according to Skorokhod (1957), (i) and (ii) imply that the distribution of $l\left(U_{n}(t)\right)$ converges to the distribution of $l(B(t))$ for any $J_{1}$-continuous functional $l$.

By the definition of $U_{n}(t)$, we have

$$
\begin{aligned}
\left|U_{n}\left(t_{1}\right)-U_{n}\left(t_{2}\right)\right| \leq & \left|\frac{\sum_{i=N\left(n t_{1}\right)+1}^{N\left(n t_{2}\right)}\left(X_{i}+Y_{i}\right)-\lambda n\left(t_{2}-t_{1}\right)\left(\mu_{F}+\mu_{G}\right)}{\left(\lambda n \kappa^{2}\right)^{1 / 2}}\right| \\
& +\left|\frac{\sum_{i=1}^{\infty} Y_{i}\left(\mathbf{1}\left(n t_{1}<T_{i} \leq n t_{2}\right)-\mathbf{1}\left(n t_{1}<T_{i}+S_{i} \leq n t_{2}\right)\right)}{\left(\lambda n \kappa^{2}\right)^{1 / 2}}\right| \\
= & : L_{1}+L_{2},
\end{aligned}
$$

with $t_{2}>t_{1}$. By Chebyshev's inequality,

$$
\mathrm{P}\left(L_{1} \geq \varepsilon\right) \leq \frac{t_{2}-t_{1}}{\varepsilon^{2}} .
$$

Furthermore,

$$
\begin{aligned}
L_{2} \leq & \left|\frac{\sum_{i=1}^{\infty} Y_{i}\left(\mathbf{1}\left(n t_{1}<T_{i} \leq n t_{2}\right)-\mathbf{1}\left(n t_{1}<T_{i} \leq n t_{2}, n t_{1}<T_{i}+S_{i} \leq n t_{2}\right)\right)}{\left(\lambda n \kappa^{2}\right)^{1 / 2}}\right| \\
& +\frac{\sum_{i=1}^{\infty} Y_{i} \mathbf{1}\left(T_{i} \leq n t_{1}, n t_{1}<T_{i}+S_{i} \leq n t_{2}\right)}{\left(\lambda n \kappa^{2}\right)^{1 / 2}} \\
= & \frac{\sum_{i=1}^{\infty} Y_{i} \mathbf{1}\left(n t_{1}<T_{i} \leq n t_{2}, T_{i}+S_{i}>n t_{2}\right)}{\left(\lambda n \kappa^{2}\right)^{1 / 2}} \\
& +\frac{\sum_{i=1}^{\infty} Y_{i} \mathbf{1}\left(T_{i} \leq n t_{1}, n t_{1}<T_{i}+S_{i} \leq n t_{2}\right)}{\left(\lambda n \kappa^{2}\right)^{1 / 2}}
\end{aligned}
$$


and, hence,

$$
\begin{aligned}
\mathrm{E}\left[L_{2}\right] \leq & \frac{\mu_{G} \sum_{i=1}^{\infty} \mathrm{P}\left(n t_{1}<T_{i} \leq n t_{2}, T_{i}+S_{i}>n t_{2}\right)}{\left(\lambda n \kappa^{2}\right)^{1 / 2}} \\
& +\frac{\mu_{G} \sum_{i=1}^{\infty} \mathrm{P}\left(T_{i} \leq n t_{1}, n t_{1}<T_{i}+S_{i} \leq n t_{2}\right)}{\left(\lambda n \kappa^{2}\right)^{1 / 2}} \\
\leq & \frac{\mu_{G} \sum_{i=1}^{\infty}\left(\mathrm{P}\left(T_{i} \leq n t_{2}<T_{i}+S_{i}\right)+\mathrm{P}\left(T_{i} \leq n t_{1}<T_{i}+S_{i}\right)\right)}{\left(\lambda n \kappa^{2}\right)^{1 / 2}} .
\end{aligned}
$$

Note that

$$
\begin{aligned}
\mathrm{P}\left(T_{i} \leq\right. & \left.n t_{2}<T_{i}+S_{i}\right)+\mathrm{P}\left(T_{i} \leq n t_{1}<T_{i}+S_{i}\right) \\
= & \int_{0}^{\infty} \lambda_{1} \mathrm{e}^{-\lambda_{1} s}\left(\mathrm{P}\left(T_{i} \leq n t_{2}<T_{i}+s\right)+\mathrm{P}\left(T_{i} \leq n t_{1}<T_{i}+s\right)\right) \mathrm{d} s \\
= & \int_{0}^{n t_{2}} \lambda_{1} \mathrm{e}^{-\lambda_{1} s} \mathrm{P}\left(T_{i} \leq n t_{2}<T_{i}+s\right) \mathrm{d} s+\int_{n t_{2}}^{\infty} \lambda_{1} \mathrm{e}^{-\lambda_{1} s} \mathrm{P}\left(T_{i} \leq n t_{2}\right) \mathrm{d} s \\
& +\int_{0}^{n t_{1}} \lambda_{1} \mathrm{e}^{-\lambda_{1} s} \mathrm{P}\left(T_{i} \leq n t_{1}<T_{i}+s\right) \mathrm{d} s+\int_{n t_{1}}^{\infty} \lambda_{1} \mathrm{e}^{-\lambda_{1} s} \mathrm{P}\left(T_{i} \leq n t_{1}\right) \mathrm{d} s,
\end{aligned}
$$

and that

$$
\begin{aligned}
\mathrm{P}\left(T_{i} \leq n t_{k}<T_{i}+s\right) & =\int_{n t_{k}-s}^{n t_{k}} \frac{\lambda^{i+1} x^{i}}{i !} \mathrm{e}^{-\lambda x} \mathrm{~d} x \\
& =\int_{0}^{s} \frac{\lambda^{i+1}\left(n t_{k}-x\right)^{i}}{i !} \mathrm{e}^{-\lambda\left(n t_{k}-x\right)} \mathrm{d} x
\end{aligned}
$$

for $k=1,2$. Let

$$
\begin{aligned}
\eta_{n}\left(t_{k}\right)= & \int_{0}^{n t_{k}} \lambda_{1} \mathrm{e}^{-\lambda_{1} s} \int_{0}^{s} \lambda \sum_{i=1}^{\infty} \frac{\lambda^{i}\left(n t_{k}-x\right)^{i}}{i !} \mathrm{e}^{-\lambda\left(n t_{k}-x\right)} \mathrm{d} x \mathrm{~d} s \\
& +\int_{n t_{k}}^{\infty} \lambda_{1} \mathrm{e}^{-\lambda_{1} s} \sum_{i=1}^{\infty} \mathrm{P}\left(T_{i} \leq n t_{k}\right) \mathrm{d} s \\
= & \int_{0}^{n t_{k}} \lambda_{1} \mathrm{e}^{-\lambda_{1} s} \int_{0}^{s} \lambda\left(1-\mathrm{e}^{-\lambda\left(n t_{k}-x\right)}\right) \mathrm{d} x \mathrm{~d} s+\lambda n t_{k} \mathrm{e}^{-\lambda_{1} n t_{k}} \\
= & \int_{0}^{n t_{k}} \lambda_{1} \mathrm{e}^{-\lambda_{1} s}\left(\lambda_{s}+\mathrm{e}^{-\lambda n t_{k}}-\mathrm{e}^{-\lambda\left(n t_{k}-s\right)}\right) \mathrm{d} s+\lambda n t_{k} \mathrm{e}^{-\lambda_{1} n t_{k}} \\
= & \lambda\left(\frac{1}{\lambda_{1}}+\left(\frac{\lambda_{1}}{\lambda\left(\lambda_{1}-\lambda\right)}-\frac{1}{\lambda_{1}}\right) \mathrm{e}^{-\lambda_{1} n t_{k}}-\frac{1}{\lambda_{1}-\lambda} \mathrm{e}^{-\lambda n t_{k}}-\frac{1}{\lambda} \mathrm{e}^{-\left(\lambda+\lambda_{1}\right) n t_{k}}\right) .
\end{aligned}
$$

From (5.3), (5.4), and (5.5), we obtain

$$
\mathrm{E}\left[L_{2}\right] \leq \frac{\mu_{G} \sum_{k=1}^{2} \eta_{n}\left(t_{k}\right)}{\left(\lambda n \kappa^{2}\right)^{1 / 2}}
$$

and, hence,

$$
\mathrm{P}\left(L_{2} \geq \varepsilon\right) \leq \frac{\mu_{G} \sum_{k=1}^{2} \eta_{n}\left(t_{k}\right)}{\varepsilon\left(\lambda n \kappa^{2}\right)^{1 / 2}} .
$$

It follows from (5.2) and (5.6) that (5.1) holds. 
Now define

$$
V_{n}(t)=\rho_{n} \lambda\left(\mu_{F}+\mu_{G}\right) n^{1 / 2} t-\left(\lambda \kappa^{2}\right)^{1 / 2} U_{n}(t) .
$$

Based on the weak convergence of $U_{n}(t)$, we obtain our final result.

Theorem 5.3. Suppose that $n^{1 / 2} \rho_{n}$ tends to a positive constant $\gamma$ as $n$ tends to $\infty$. Then $V_{n}(t) \stackrel{\mathrm{W}}{\rightarrow} V(t)$, where

$$
V(t)=\gamma \lambda\left(\mu_{F}+\mu_{G}\right) t-\left(\lambda \kappa^{2}\right)^{1 / 2} B(t) .
$$

Furthermore, for any positive constants $u$ and $\delta$,

$$
\lim _{n \rightarrow \infty} \psi\left(n^{-1 / 2} \delta, n^{1 / 2} u\right)=\mathrm{e}^{-2 \delta u}
$$

where $\psi\left(n^{-1 / 2} \delta, n^{1 / 2} u\right)$ is the ultimate ruin probability for model (2.1) with initial surplus $n^{1 / 2} u$ and security loading $n^{-1 / 2} \delta$.

Proof. The weak convergence of $V_{n}(t)$ is simply due to Theorem 5.1 and the limit theorem of Skorokhod (1957). Result (5.7) follows from Grandell (1978).

\section{Acknowledgements}

The first author was supported by a grant from the Research Grants Council of the Hong Kong Special Administrative Region, China (project no. HKU 7202/99H) and the second author was supported by the National Natural Science Foundation of China (grant no. 10271062).

\section{References}

Boogaert, P. And Haezendonck, J. (1989). Delay in claim settlement. Insurance Math. Econom. 8, 321-330.

Brémaud, P. (2000). An insensitivity property of Lundberg's estimate for delayed claims. J. Appl. Prob. 37, 914-917.

DufFIELd, N. G. AND O'ConNell, N. (1995). Large deviations and overflow probabilities for the general single-server queue, with applications. Math. Proc. Camb. Phil. Soc. 118, 363-374.

Grandell, J. (1977). A class of approximations of ruin probabilities. Scand. Actuarial J., 37-52.

Grandell, J. (1978). A remark on: ‘A class of approximations of ruin probabilities'. Scand. Actuarial J., 77-78.

Iglehart, D. L. (1969). Diffusion approximations in collective risk theory. J. Appl. Prob. 6, 285-292.

SковокноD, A. V. (1957). Limit theorems for stochastic processes with independent increments. Theory Prob. Appl. 2, 138-171.

Waters, H. R. and Papatriandafylou, A. (1985). Ruin probabilities allowing for delay in claims settlement. Insurance Math. Econom. 4, 113-122.

Yuen, K. C. AND GuO, J. Y. (2001). Ruin probabilities in the binomial model with time-correlated aggregate claims. Insurance Math. Econom. 29, 47-57. 